\title{
Potent Inhibition of Severe Acute Respiratory Syndrome Coronavirus 2 (SARS-CoV-2) by photosensitizers
}

\section{Shujuan Yu}

Fuzhou University

\section{Gaohui Sun}

Fuzhou University

Yaqun Sui

Fuzhou University

\section{Hanlin Li}

Fuzhou University

\section{Ning Zhang}

Institute of Microbiology, Chinese Academy of Sciences

\section{Yuhai Bi}

Institute of Microbiology, Chinese Academy of Sciences

\section{George Gao}

Institute of Microbiology, Chinese Academy of Sciences

\section{Longguang Jiang}

Fuzhou University

\section{Peng Xu}

Fuzhou University

\section{Cai Yuan}

Fuzhou University

\section{Yang Yang}

Shenzhen Third People's Hospital, Second Hospital Affiliated to Southern University of Science and Technology

Mingdong Huang (D HMD_lab@fzu.edu.cn)

Fuzhou University https://orcid.org/0000-0003-0085-9141

\section{Research Article}

Keywords: COVID-19, SARS-CoV-2 inactivation, photodynamic therapy, photosensitizer

Posted Date: February 8th, 2021 
DOI: https://doi.org/10.21203/rs.3.rs-152985/v1

License: (c) (1) This work is licensed under a Creative Commons Attribution 4.0 International License. Read Full License 


\section{Abstract}

The pandemic of coronavirus disease 2019 (COVID-19) caused by severe acute respiratory syndrome coronavirus 2 (SARS-CoV-2) has exploded since December 2019, and causes more than 2 million death with more than 95 million people infected as of Jan. 21th, 2021 globally1,2. Angiotensin-converting enzyme 2 (ACE2), expressed in the lungs, arteries, heart, kidney, intestines, and nasal epithelium3, has been shown to be the primary entry point targeted by the surface spike protein of SARS-CoV-2. Currently, no proven antiviral treatment for SARS-CoV-2 infection is available. In this study, we screened a number of photosensitizers for photodynamic viral inactivation, and found compounds pentalysine $\beta$ carbonylphthalocyanine zinc (ZnPc5K) and chlorin e6 (ce6) potently inhibited the viral infection and replication in vitro with half-maximal effective concentrations (EC50) values at nanomolar level. Such viral inactivation strategy is implementable, and has unique advantages, including resistance to virus mutations, affordability compared to the monoclonal antibodies, and lack of long-term toxicity.

\section{Background}

Firstly, we established a facile pseudoviral entry assay. In this system, the pseudovirus containing the spike (S) protein of SARS-CoV-2 and EGFP protein encapsulated on HIV viral capsid (Fig.1a) efficiently infected the human ACE2 expressing 293T cells (ACE2-293T) but not the plain 293T cells (Fig.1b and Fig.S1a), indicating that the observed infection was mediated by the molecular interaction between the spike and ACE2 proteins. The infected ACE2-293T cells adhered to the microplate and proliferated normally up to 7 days. Bright green fluorescence of EGFP appeared at about 12 hours post infection (h.p.i), increased as cell proliferated, and maximized at about 48 h.p.i. The fluorescence intensity is proportional to amount of pseudoviruses added to ACE2-293T cells.

Then, we screened a number of representative photosensitizers for their antiviral effects using the pseudoviral entry assay. The photosensitizers (Fig.1C) were mixed with the pseudoviruses at the indicated concentrations and illuminated with a light-emitting diode (LED) light source to a light dose of 0.48 $\mathrm{J} / \mathrm{cm}^{2}$, then the mixture was incubated with ACE2-293T cells for $48 \mathrm{hr}$, followed by image analysis on a high-content imager. Two photosensitizers (Fig.1c, ZnPc5K and ce6) were identified to completely abolish the infection of the pseudoviruses to the host cells at doses of $1.0 \mu \mathrm{M}$ and with LED light illumination (Fig.1C). Importantly, these two photosensitizers did not affect the cell proliferation, adhesion, and morphology at this concentration even under the light illumination (Fig.S1b). As a control, the photosensitizers did not affect the infection under the otherwise identical condition in the absence of LED light illumination (Fig.S1c). Furthermore, we measured the inhibitory potencies of the two photosensitizers, and identified the EC50 values of $6.2 \mathrm{nM}$ and $177 \mathrm{nM}$ for ZnPc5K and ce6, respectively (Fig.1c). Meanwhile, the $90 \%$ effective concentrations ( $\mathrm{EC}_{90}$ ) values were $114 \mathrm{nM}$ and $515 \mathrm{nM}$ for $\mathrm{ZnPc} 5 \mathrm{~K}$ and ce6, respectively (Fig.1c). We then studied if the photodynamic effect could inactivate the pseudovirus inside the ACE2-293T cells. We incubated the pseudoviruses with the ACE2-293T cells and photosensitizers for $4 \mathrm{hr}$ to allow viral infection to the cells, followed by LED illumination $\left(0.48 \mathrm{~J} / \mathrm{cm}^{2}\right)$. The cells were allowed to continue to grow for 2 days, followed by image analysis on a high-content 
imager. The results showed that both photosensitizers reduced the green fluorescence signal of the cells in a dose dependent manner (Fig.1d). For ZnPc5K, the pseudoviral fluorescence signal was reduced by $\sim 40 \%$ at a dose of $740 \mathrm{nM}$, while only about $20 \%$ reduction of pseudoviral fluorescence signal for ce6 was observed. Moreover, both photosensitizers showed little toxicity to the infected host cells as detected by nucleus stain (Fig.S1d).

To further confirm the antiviral efficiency of the two compounds, we used live SARS-CoV-2 to test the antiviral activity in vitro. The photosensitizers (ZnPc5K or ce6) were incubated with SARS-CoV-2 viruses, followed by LED light illumination $\left(0.48 \mathrm{~J} / \mathrm{cm}^{2}\right)$. The mixture was then allowed to infect the Vero cells for 2 days, and the inactivation rates were evaluated by quantification of viral copy numbers in the cell supernatant via quantitative RT-PCR (qRT-PCR) and further confirmed with immunofluorescence staining (Fig.2a, b). Similar results with the pseudoviral entry assay were found. ZnPc5K and ce6 efficiently inhibited the virus with $\mathrm{EC}_{50}$ values of $177 \mathrm{nM}$ and $156 \mathrm{nM}$, and $\mathrm{EC}_{90}$ values of $308 \mathrm{nM}$ and $352 \mathrm{nM}$, respectively. Meanwhile, photosensitizers had no effect on SARS-CoV-2 viruses under the identical conditions without LED illumination (Fig.S2). Upon ZnPc5K or ce6 treatments with illumination, Vero cells were shown almost no phototoxicity at concentration lower than $5 \mu \mathrm{M}$ (Fig.2c). Next, we studied the photodynamic effect on the viruses in the host cells. The live virus and photosensitizers were mixed and added to the Vero cells for $4 \mathrm{hr}$ incubation at $37^{\circ} \mathrm{C}$. The cell-containing mixtures were illuminated with LED once and allowed to continue to grow for $44 \mathrm{hr}$ before qRT-PCR viral quantification. The results showed that $\mathrm{ZnPc5K}$ and ce6 reduced the virus load by $60 \%$ and $10 \%$, respectively, at $50 \mathrm{nM}$ concentration (Fig.S3). The ce6 required higher concentration (100 nM) to reduce the virus load by $50 \%$. The host cells maintained normal morphology during photodynamic treatment (Fig.S3), suggesting no toxicity at these concentrations. Cytotoxicity was further studied on fibroblast (HELF cells) and ACE2293T cell lines, and ZnPc5K showed no phototoxicity at concentration lower than $5 \mu \mathrm{M}$ (Fig.S4), while ce6 showed no phototoxicity at concentration lower than $1 \mu \mathrm{M}$ (Fig.S4).

Here we demonstrate that photodynamic method is possible to inactivate SARS-CoV-2 outside and within the host cells without toxicity. Such specificity of photosensitizer is likely due to the detoxifying capability of the cells, but not the virus. The cells contain a number of enzymes like superoxide dismutase and catalase, which can remove the reactive oxygen species (ROS) generated by the photosensitizers, making them more resilient to the insults from photosensitizers. ZnPc5K showed high antiviral efficacy while maintaining no toxicity to cells. This photosensitizer has low toxicity (acute oral toxicity $>5000 \mathrm{mg} / \mathrm{kg}$ ) with no irritation to eyes or skins, and causes no mutagenesis to cells ${ }^{4}$. It has been demonstrated to be effective in antibacterial and anti-tumor cells in many studies. Generally, phthalocyanine type compound is quite safe and has been used as a color dye for outfits and underwear in fabric industry for over a century. Phthalocyanine type compound has also been used as an anti-tumor drug (Photosense ${ }^{\circledR}$ ) for cancer treatment in Ukraine since $1990 s^{5}$. Another phthalocyanine-based compound (Photocyanine ${ }^{\circledR}$ ) is currently under Phase II clinical trial for esophagus cancer with a dose of $0.05-0.1 \mathrm{mg} / \mathrm{kg}$ in China ${ }^{6,7}$. Its Phase I trial demonstrated no major adverse effect in human at a dose of $2 \mathrm{mg} / \mathrm{kg}$. Phthalocyanine green has been approved by FDA ${ }^{8}$ to use in contact lens, surgical suture, and latex condom as a color additive. 
Phthalocyanine was also approved by FDA as Indirect Additives used in food contact substances. A surprised finding from this work is the high potency of $\mathrm{ZnPc5K}$ to inactivate virus with an $\mathrm{EC}_{50}$ of $177 \mathrm{nM}$, much higher than the typical micromolar range potencies for antimicrobial or antitumor applications of photosensitizers $^{9-11}$. A number of studies found that photosensitizer-like structure with large ring-like organic compound, including methylene blue ${ }^{12}$ and heme ${ }^{13,14}$, may have specific binding to SARS-CoV-2 virus $^{15}$.

Photosensitizer requires light illumination to be effective for virus inactivation How to deliver light? There a couple scenarios for the applications of photosensitizers to inactivate virus ${ }^{15,12,16}$ : (1) The SARSCoV-2 virus entry receptors (ACE2 and TMPRSS2) are highly expressed in the nasal epithelium ${ }^{3}$, and the virus was found to propagate in the upper respiratory tract, especially in the nose and oropharynx. The photodynamic treatment can reduce the number of virus present in the upper respiratory tract.

Photosensitizer can be delivered directly by nebulization, following by LED light illumination. (2) The red light can be delivered through endoscope into the lung, which is relatively transparent to red light compared to other organs. In combination with photosensitizer, infused through vein, virus inside the lung can be inactivated, helping to reduce the burden of immune system. Such invasive procedure can be used in intensive care unit.

The current method of virus inactivation in our study has several advantages. First, this method works independent of viral sequences, and resistant to virus mutations. Photosensitizers use ROS to damage viral envelope proteins and/or nucleic acids. Such mechanism is most likely not sensitive to the mutations of SARS-CoV-2, which has been reported, e.g., D614G of the spike protein ${ }^{17}$. Second, the debris or the fragments generated from the virus by ROS could stimulate host immune defense. Third, photodynamic method to inactivate virus can be more affordable than other therapeutics like monoclonal antibody, which can be an important factor for preventive use at home. Other advantages of photodynamic inactivation include the lack of long-term toxicity, the ability to remove virus in a very short time, less damage to adjacent tissues, and high repeatability without viral resistance.

In summary, we demonstrated the high potencies (nM of EC50) of photosensitizers in the inactivation of SARS-CoV-2 virus. Importantly, the method can also reduce the virus load inside the human host cells with a large safety margin to cells.

\section{Materials And Methods}

\section{Chemicals and reagents}

ZnPc5K was synthesized accordingly to our previously published procedure ${ }^{18}$. ce6 was from Cayman Chemical. Polybrene was from Sigma-Aldrich, USA. Puromycin was from Invitrogen, USA. Oligo nucleotides were synthesized by Sangon Biotech, China.

\section{Cells and Viruses}


293T and Vero cells negative for mycoplasma were obtained from ATCC and cultured at $37^{\circ} \mathrm{C}$ with $5 \%$ $\mathrm{CO}_{2}$ in Dulbecco's Modified Eagle medium (DMEM) supplemented with 10\% FBS (Gibco) and 1x penicillinstreptomycin (Invitrogen). SARS-CoV-2 pseudovirus was purchased from Genewiz, China. SARS-CoV-2 strain BetaCoV/Shenzhen/SZTH-003/2020 (GISAID No. EPI_ISL_406594) was isolated and stored in the bio-safety level 3 laboratory in Shenzhen Third People's Hospital.

\section{Lentivirus packaging and stable cell line construction}

The pLVX-hACE2 plasmid was constructed by cloning the coding region from Human ACE2 CDNA ORF Clone with N-terminal Flag tag (SinoBiological, HG10108-NF, China) into pLVX-IRES-Puro Lentiviral vector between Xhol and Notl sites.

Lentivirus was packaged by co-transfection of pLVX-hACE2 or empty vector, with a mixture of VSV-G (Addgene, 8454), PRRE (Addgene, 12251) and REV (Addgene, 12253) plasmids by FuGENE (Promega) into $293 \mathrm{~T}$ cells. The transfected medium was changed into fresh DMEM $8 \mathrm{hr}$ later. After $48 \mathrm{hr}$ of transfection, the lentivirus-containing supernatants were collected and filtered through a $0.45-\mu \mathrm{m}$ filter, and diluted 1:1 with fresh medium containing $8 \mu \mathrm{g} / \mathrm{ml}$ polybrene, and were used to infect the target cells (293T) grown at 70 80\% confluence. The selection antibiotic puromycin was added at a killing concentration 1-2 $\mu \mathrm{g} / \mathrm{mL}$ to select cell line stably expressing ACE2. Fresh media with antibiotic were added every two days until all the cells in the control wells were dead.

\section{Infection assay of SARS-CoV-2 pseudovirus}

Either plain 293T cells or ACE2-293T cells (4000 per well) were seeded in CellCarrier-384-well microplate (PerkinElmer) on day 0. SARS-CoV-2 pseudovirus $\left(0.4 \times 10^{5}\right.$, i.e. $\left.\mathrm{MOI}=10\right)$ was then added to the cells on day 1. From then on, EGFP and bright field images of these wells were taken by Operetta High Content Imaging System every 24 hr till day 8.

\section{Inactivation assay of SARS-CoV-2 pseudovirus by photosensitizers}

Pseudovirus inactivation assay was performed by mixture of SARS-CoV-2 pseudovirus with serially diluted photosensitizers (such as ZnPc5K and ce6) with or without the light exposure using a LED light source (SunDynamic, Inc., Qingdao, $4 \mathrm{~mW} / \mathrm{cm}^{2}$ ) of $660 \mathrm{~nm}$ for $2 \mathrm{~min}$ (to a light dosage of $0.48 \mathrm{~J} / \mathrm{cm}^{2}$ ), followed by addition of the mixture into ACE2-293T cells. At 48 h.p.i, the fluorescence images were taken and analyzed on Operetta High Content Imaging System (Perkin Elmer). The relative viral infectivity was proportional to the sum of EGFP spot intensity on the cells and was analyzed by Operetta CLS software. Standard errors were estimated based on duplicates of the experiments.

Pseudovirus cellular inactivation assay was carried out by mixing SARS-CoV-2 pseudovirus with serially diluted photosensitizers. The mixture was added into ACE2-293T cells, and $4 \mathrm{hr}$ later illuminated by LED with a power of $4 \mathrm{~mW} / \mathrm{cm}^{2}$ for $2 \mathrm{~min}$. After incubation in $\mathrm{CO}_{2}$ incubator for $48 \mathrm{hr}$, images (EGFP for virus 
and Hoechst33342 for nucleus stain) were taken by Operetta High Content Imaging System to detect EGFP intensity.

An automated analysis method was used based on Operetta CLS ${ }^{\mathrm{TM}}$ System. To measure bioactivity of SARS-CoV-2 pseudovirus, we calculated the sum of EGFP fluorescent intensity for pseudovirus extracellular inactivation assay and the mean of EGFP fluorescent intensity for pseudovirus cellular inactivation assay. For such analysis, we first defined nucleus with Hoechst-staining images, then cytoplasm with bright field images, and finally calculated the EGFP fluorescent intensity by going through all EGFP spots inside cells. The virus inactivation percentage was calculated by subtracting the intensity of EGFP of each group from the control of no photosensitizer, and divided by the control fluorescence. Error bars represent the SD of triplicates in one experiment.

\section{Evaluation of antiviral activities of the photosensitizers using the live virus}

Firstly, the titer of the viral stock was measured by $50 \%$ tissue culture infective dose $\left(\mathrm{TCID}_{50}\right)$, then the antiviral activities of the photosensitizers were evaluated as previously reported with some modifications ${ }^{19}$. Vero cells were seeded in a 24 -well transparent plate $24 \mathrm{hr}$ before infection. On the day of infection, the cells were washed twice with PBS. The SARS-COV- 2 with a dose of 0.02 multiplicity of infection (MOI) was mixed with serially diluted photosensitizer (at the concentration of $0.001 \mu \mathrm{M}, 0.01$ $\mu \mathrm{M}, 0.05 \mu \mathrm{M}, 0.1 \mu \mathrm{M}, 0.5 \mu \mathrm{M}, 1 \mu \mathrm{M}$ or $5 \mu \mathrm{M})$ in cell culture medium without or with the light exposure using a LED light source of $660 \mathrm{~nm}$ and with a power of $0.48 \mathrm{~J} / \mathrm{cm}^{2}$. This mixture $(500 \mu \mathrm{L})$ was added to the Vero cells and further cultured with fresh DMEM with $2 \%$ FBS at $37^{\circ} \mathrm{C}$. At 48 th h.p.i, the cell supernatant was collected and viral RNAs were extracted using the QIAamp RNA Viral Kit (Qiagen, Heiden, Germany) for further quantification analysis. The cells were collected for immunofluorescence assay. All the experiments involving infectious SARS-CoV-2 were handled in BSL-3 facilities at the Shenzhen Third People's Hospital.

\section{Quantitative real-time PCR ${ }^{20}$}

Viral RNAs were extracted from the samples using the QIAamp RNA Viral Kit (Qiagen, Heiden, Germany), and qRT-PCR was performed using a commercial kit (Genrui-bio) targeting the $\mathrm{S}$ and $\mathrm{N}$ genes. The specimens were considered positive if the $\mathrm{Ct}$ value was less than 38.0, and negative if the results were undetermined. Specimens with a $\mathrm{Ct}$ higher than 38 were repeated. The specimen was considered positive if the repeat results were the same as the initial result and between 38 and 40 . If the repeat $\mathrm{Ct}$ was undetectable, the specimen was considered negative.

\section{Immunofluorescence assay ${ }^{19}$}

Vero cells were fixed in $4 \%$ formaldehyde at 48 th h.p.i. Then cells were permeabilized in $0.5 \%$ Triton X100 , blocked in 5\% BSA in PBS, and then probed with the plasma of this patient or healthy control at a dilution of 1:500 for $1 \mathrm{~h}$ at room temperature. The cells were washed three times with PBS and then incubated with either goat anti-human IgG conjugated with Alexa fluor 488 at a dilution of 1:500 for $1 \mathrm{~h}$ 
(Invitrogen). The cells were then washed and stained with Hoechest33342 (Invitrogen) to detect nuclei. Fluorescence images were obtained and analyzed using EVOS FL Auto Imaging System (Invitrogen).

\section{CCK-8 assay}

The cell viability/cytotoxicity was measured by Cell Counting Kit-8 (CCK-8) (Meilunbio). Briefly, the cells were seeded in ViewPlate-96-well plate (Perkin Elmer), and treated with serially diluted photosensitizers, followed by LED illumination with a power of $4 \mathrm{~mW} / \mathrm{cm}^{2}$ for $2 \mathrm{~min}$ at $4 \mathrm{hr}$ later. After incubation in $\mathrm{CO} 2$ incubator for $24 \mathrm{hr}, 10 \mu \mathrm{l}$ of CCK-8 solution was added to each well, and the 96 -well plate was incubated at $37^{\circ} \mathrm{C}$ for $1 \mathrm{hr}$. Normal DMEM with CCK-8 solution and the cells with no photosensitizer served as the blank control and normal control, respectively. The cell viability was calculated according to the product manufacturer's instructions using the OD450nm measured on a microplate reader (SpectraMax ${ }^{\circledR} \mathrm{i} 3 \mathrm{x}$, Molecular Devices). All doses were done at triplicates.

\section{Declarations}

\section{Acknowledgements:}

Our research work is financially supported by grants from National Key R\&D Program of China (2017YFE0103200), Natural Science Foundation of China (31670739, 22077016, 82070142, 31870163), Strategic Priority Research Program of the Chinese Academy of Sciences (CAS) (Grant No. XDB29010102), the NSFC Outstanding Young Scholars (Grant No. 31822055), and Youth Innovation Promotion Association of CAS (Grant No. 2017122).

\section{References}

1 WHO Coronavirus Disease (COVID-19) Dashboard, <https://covid19.who.int/> (2020).

2 Wiersinga, W. J., Rhodes, A., Cheng, A. C., Peacock, S. J. \& Prescott, H. C. Pathophysiology, Transmission, Diagnosis, and Treatment of Coronavirus Disease 2019 (COVID-19): A Review. JAMA 324, 782-793, doi:10.1001/jama.2020.12839 (2020).

3 Sungnak, W. et al. SARS-CoV-2 entry factors are highly expressed in nasal epithelial cells together with innate immune genes. Nature Medicine 26, 681-687, doi:10.1038/s41591-020-0868-6 (2020).

4 Ying, C. et al. Safety evaluation of an amino acid based photosensitizer (in Chinese). J Toxicol 29, 477-479, doi:10.16421/j.cnki.1002-3127.2015.06.021 (2015).

5 Smirnova, Z. S. et al. Efficiency and Pharmacokinetics of Photosense: A New Liposomal Photosensitizer Formulation Based on Aluminum Sulfophthalocyanine. Pharmaceutical Chemistry Journal 39, 341-344, doi:10.1007/s11094-005-0150-8 (2005). 

cancer treatment. Journal of Innovative Optical Health Sciences 13, 2030009, doi:10.1142/s1793545820300098 (2020).

$7 \quad \mathrm{Li}$, S. et al. A phase I study to evaluate the application of photocyanine using pharmacokinetic and pharmacodynamic analysis in patients with malignancy. Cancer chemotherapy and pharmacology 86, 267-276, doi:10.1007/s00280-020-04096-y (2020).

8 Summary of Color Additives for Use in the United States in Foods, Drugs, Cosmetics, and Medical Devices, <https://www.fda.gov/industry/color-additive-inventories/summary-color-additives-use-unitedstates-foods-drugs-cosmetics-and-medical-devices> (2020).

9 Chen, Z. et al. Photodynamic antimicrobial chemotherapy using zinc phthalocyanine derivatives in treatment of bacterial skin infection. J Biomed Opt 21, 18001, doi:10.1117/1.JB0.21.1.018001 (2016).

10 Chen, Z. et al. Pentalysine beta-carbonylphthalocyanine zinc: an effective tumor-targeting photosensitizer for photodynamic therapy. ChemMedChem 5, 890-898, doi:10.1002/cmdc.201000042 (2010).

11 Chen, Z. et al. An effective zinc phthalocyanine derivative for photodynamic antimicrobial chemotherapy. Journal of Luminescence 152, 103-107, doi:10.1016/j.jlumin.2013.10.067 (2014).

12 Gendrot, M. et al. Methylene blue inhibits replication of SARS-CoV-2 in vitro. International Journal of Antimicrobial Agents, 106202, doi:10.1016/j.ijantimicag.2020.106202 (2020).

13 Liu, W. \& Li, H. Preprint at https://doi.org/10.26434/. chemrxiv.11938173.v9(2020).

14 Read, R. J. Preprint at https://doi.org/10.26434/. chemrxiv.12120912.v1 (2020). (2020).

15 Kipshidze, N., Yeo, N. \& Kipshidze, N. Photodynamic therapy for COVID-19. Nature Photonics 14, 651-652, doi:10.1038/s41566-020-00703-9 (2020).

16 Dias, L. D., Blanco, K. C. \& Bagnato, V. S. COVID-19: Beyond the virus. The use of photodynamic therapy for the treatment of infections in the respiratory tract. Photodiagnosis and photodynamic therapy 31, 101804-101804, doi:10.1016/j.pdpdt.2020.101804 (2020).

17 Plante, J. A. et al. Spike mutation D614G alters SARS-CoV-2 fitness. Nature, doi:10.1038/s41586020-2895-3 (2020).

18 Jincan, C. et al. Synthesis and Photodynamic Activity of a New Type of Pentalysine 2Carbonylphthalocyanine Zinc (in Chinese). Chemical Journal of Chinese Universities 29, 2131-2137 (2008). 
19 Zhang, H. et al. A novel virtual screening procedure identifies Pralatrexate as inhibitor of SARSCoV-2 RdRp and it reduces viral replication in vitro. PLoS computational biology 16, e1008489, doi:10.1371/journal.pcbi.1008489 (2020).

20 Zhao, F. et al. The Time Sequences of Respiratory and Rectal Viral Shedding in Patients With Coronavirus Disease 2019. Gastroenterology 159, 1158-1160.e1152, doi:10.1053/j.gastro.2020.05.035 (2020).

\section{Figures}




\section{Figure 1}

(a)

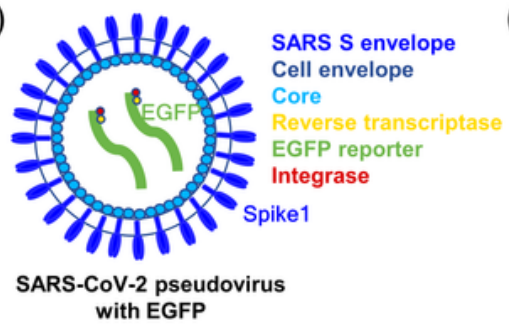

(c)

(b)

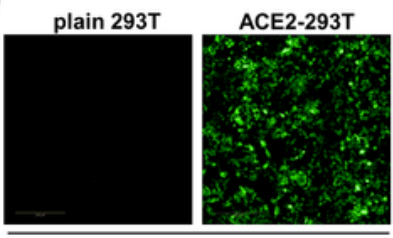

SARS-CoV-2 pseudovirus

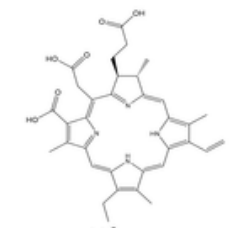

ce6
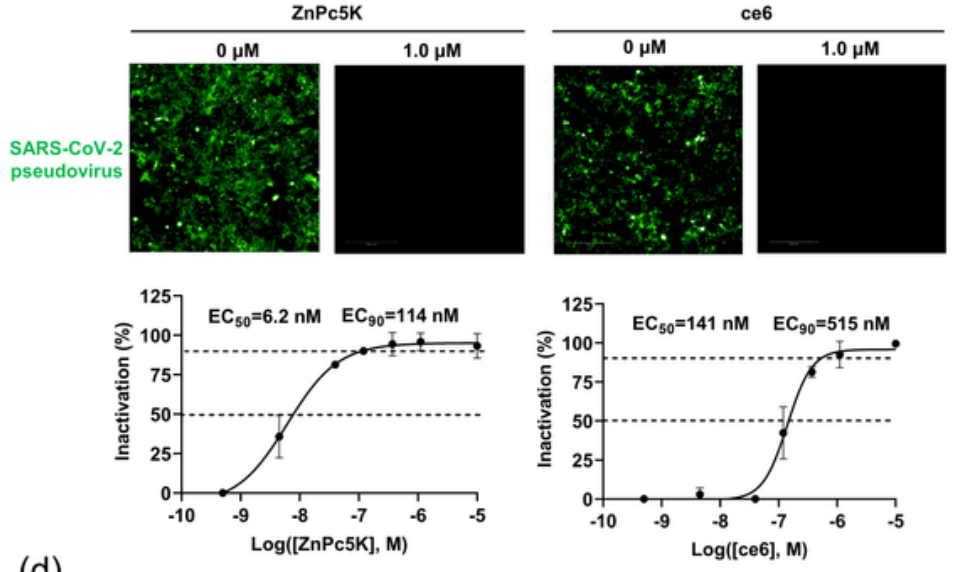

(d)

Black plate, $0.48 \mathrm{~J} / \mathrm{cm}^{2}$

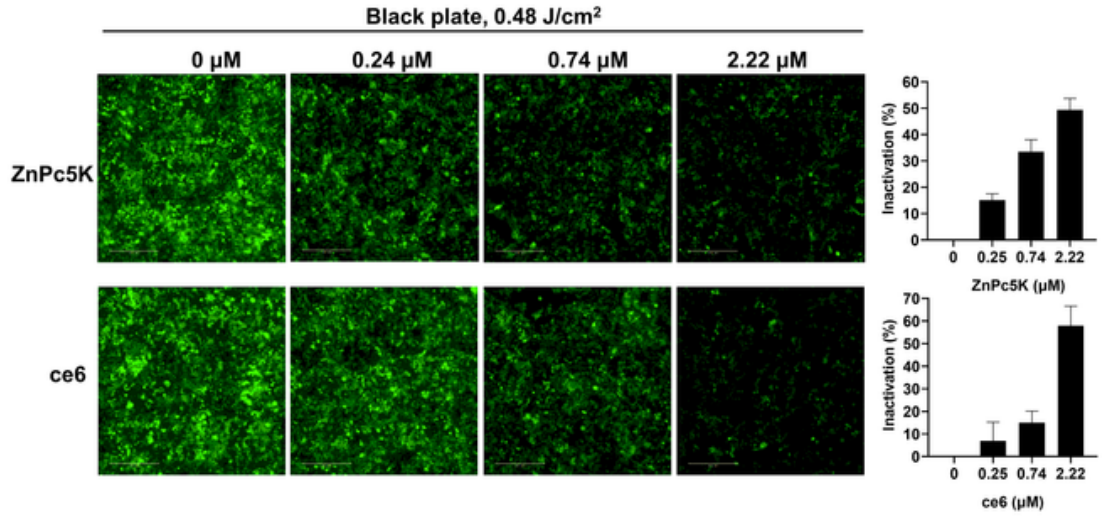

Figure 1

Screening of two photosensitizers by SARS-CoV-2 pseudo- viruses entry assay . A pseudovirus containing SARS-CoV-2 spike protein on the envelope of a lentiviral core and EGFP (a, 0.4x105, i.e. MOI=10) infected ACE2-293T but not the plain 293T cells, giving strong fluorescence of EGFP (b). (c) Photosensitizer ZnPc5K and ce6 (molecular structures were shown here) completely inhibited the viral infectivity at $1 \mu \mathrm{M}$ concentration upon LED illumination. Dose-responses of the inhibition of viral infectivity were also 
measured for the photosensitizers, giving the EC50 and EC90. The EGFP intensities of all fields in a microplate well at different concentrations were summed to represent the virus infected into the cells. Error bars represent the SD of duplicates in one experiment. Scale bar, $500 \mu \mathrm{m}$. (d) Photosensitizers inactivated the SARS-CoV-2 pseudoviruses and reduced virus load inside ACE2-293T cells upon LED illumination. Serially diluted ZnPc5K or ce6 and pseudoviruses were incubated with ACE2-293T cells for 4 $\mathrm{hr}$, and illuminated by LED to a light dose of $0.48 \mathrm{~J} / \mathrm{cm} 2$. After further $48 \mathrm{hr}$ incubation, the fluorescent images (green for EGFP for virus and blue for Hoechst for nucleus staining) were recorded and analyzed. Error bars represent the SD of triplicates in one experiment. Scale bar, $500 \mu \mathrm{m}$. 


\section{Figure 2}

(a)
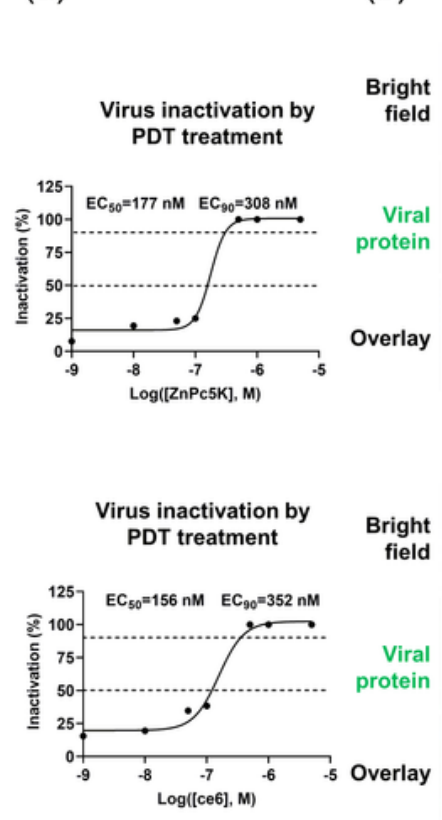

(b)
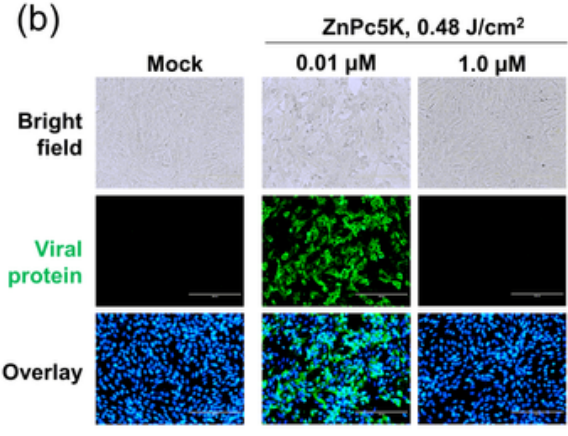

ce6, $0.48 \mathrm{~J} / \mathrm{cm}^{2}$

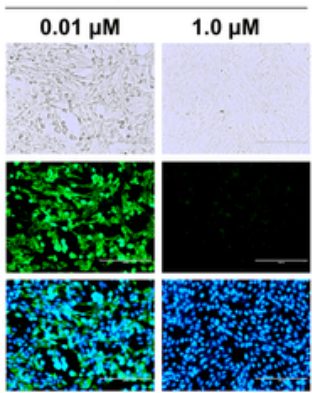

(c)

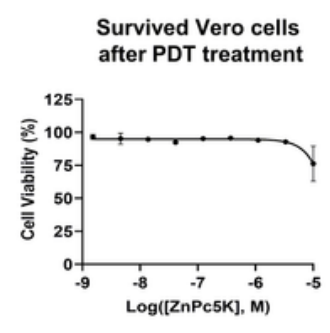

Survived Vero cells after PDT treatment

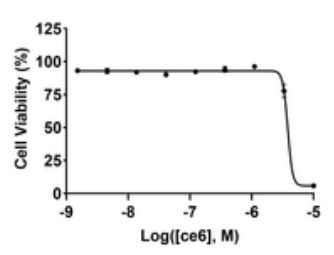

\section{Figure 2}

Photosensitizers ZnPc5K and ce6 effectively inactivated SARS-CoV-2 live virus upon LED illumination. (a) Dose response curve of viral inactivation upon photodynamic treatment. The SARS-COV-2 with a dose of 0.02 multiplicity of infection (MOI) was incubated with serially diluted photosensitizer and illuminated, followed by addition to Vero cells and further incubation for $48 \mathrm{hr}$. The viral yield in the cell supernatant was quantified by qRT-PCR. (b) Immunofluorescence microscopic images of virus infection upon 
treatment of $\mathrm{ZnPc5K}$ and ce6 at the indicated concentrations with illumination showed the efficacy of photosensitizers to virus and safety to host cells. The fluorescence images were taken at 48 h.p.i. Scale bar, $200 \mu \mathrm{m}$. Cells were stained with the viral protein (green) and nuclei (blue). (c) Cytotoxicity of ZnPc5K or ce6 to Vero cells was measured by CCK-8 assay.

\section{Supplementary Files}

This is a list of supplementary files associated with this preprint. Click to download.

- SI20210122.docx 